\title{
Ablation of typical atrial flutter as therapeutic component in carcinoid heart disease: a case report
}

\author{
Susann Groschke, RolfWeinert, Björn Becker, Gert Richardt, Ralph Tölg, Leon Iden and Martin Borlich* (1)
}

\begin{abstract}
Background: Carcinoid heart disease is the cardiac manifestation of carcinoid syndrome. There is limited research on rhythm management in patients with carcinoid heart disease. The association of typical atrial flutter and carcinoid heart disease in particular is poorly described.

Case presentation: Here we present a case of a 77-year-old German woman with carcinoid heart disease and recurrent typical atrial flutter complicating the postoperative course after tricuspid valve replacement and its successful long-term rhythm control by ablation therapy.

Conclusion: There is limited evidence on rhythm management in patients with the rare diagnosis of carcinoid heart disease. Typical atrial flutter repeatedly complicated the postoperative course of our patient with carcinoid heart disease and could finally be treated curatively by ablation. Radiofrequency ablation should be considered as a valuable therapeutic component in the challenging therapy of this disease.
\end{abstract}

Keywords: Carcinoid heart disease, Hedinger syndrome, Ablation, Typical atrial flutter, Neuroendocrine tumor

\section{Background}

Carcinoid heart disease (CHD) is a rare and challenging organ manifestation of neuroendocrine tumors (NET) caused by fibrotic remodeling processes, especially of the right ventricular endocardium, and is associated with a poor prognosis. Differentiated NET, together with poorly differentiated neuroendocrine carcinomas (NEC), belongs to the group of neuroendocrine neoplasms (NEN). Although well-differentiated, low-proliferative NET may be found incidentally in pathology specimens, some hormone-active NET lead to physical discomfort caused by the release of bioactive peptides. Carcinoid syndrome is the most common functionally active NET, in which tumor cells release large amounts

*Correspondence: martin.borlich@segebergerkliniken.de

Heart Center, Segeberger Kliniken GmbH (Academic Teaching Hospital of the Universities of Kiel, Lübeck and Hamburg), Am Kurpark 1,

23795 Bad Segeberg, Schleswig-Holstein, Germany of serotonin, tachykinins and transforming growth factor beta (TGF- $ß$ ), resulting in flush, diarrhea, and abdominal pain. Carcinoid tumors are predominantly found in the gastrointestinal tract, the bronchial system, and the urogenital tract [1]. In the case of progressive tumor growth with liver metastases and increased release of vasoactive substances, the hepatic first-pass effect gets lost, and the heart is exposed to increasing hormone concentrations that lead to the development of carcinoid heart disease, also known as Hedinger syndrome. The increasing hormone concentrations inside the heart lead to the deposition of fibrous plaques on the endocardium of the right-sided heart valves and ventricles, which can result in right heart failure that is difficult to treat. Little is known about the occurrence of arrhythmias in patients with CHD. Besides rarely occurring atrial fibrillation and ventricular tachycardia, typical atrial flutter has been reported only once as the initial symptom of carcinoid syndrome and CHD. We describe a case in which typical original author(s) and the source, provide a link to the Creative Commons licence, and indicate if changes were made. The images or other third party material in this article are included in the article's Creative Commons licence, unless indicated otherwise in a credit line to the material. If material is not included in the article's Creative Commons licence and your intended use is not permitted by statutory regulation or exceeds the permitted use, you will need to obtain permission directly from the copyright holder. To view a copy of this licence, visit http://creativecommons.org/licenses/by/4.0/. The Creative Commons Public Domain Dedication waiver (http://creativeco mmons.org/publicdomain/zero/1.0/) applies to the data made available in this article, unless otherwise stated in a credit line to the data. 
atrial flutter repeatedly complicated the postoperative course of a patient with CHD and could be treated definitively by ablation.

\section{Case presentation}

A 77-year-old German woman presented to our emergency department with dyspnea and peripheral edema, which was first noted a few days ago. Previous ultrasound had ruled out deep vein thrombosis (DVT), and diuretic therapy had already been initiated leading to a slight alleviation of her symptoms. The medical history included tonsillectomy, appendectomy, and hysterectomy. No other previous diseases were known at that time. On physical examination, we noted a marked jugular vein pulse and discrete edema of the lower extremities. Pulmonary and cardiac auscultation revealed no abnormal findings. Electrocardiography (ECG) showed sinus rhythm with a heart rate of 95 beats per minute without further abnormalities. On transthoracic echocardiography we noticed a dilated right heart with severe tricuspid regurgitation (Fig. 1). No other cardiac defects were seen; the left ventricular systolic function was normal. Using continuous wave $(\mathrm{CW})$ Doppler analysis for estimating the
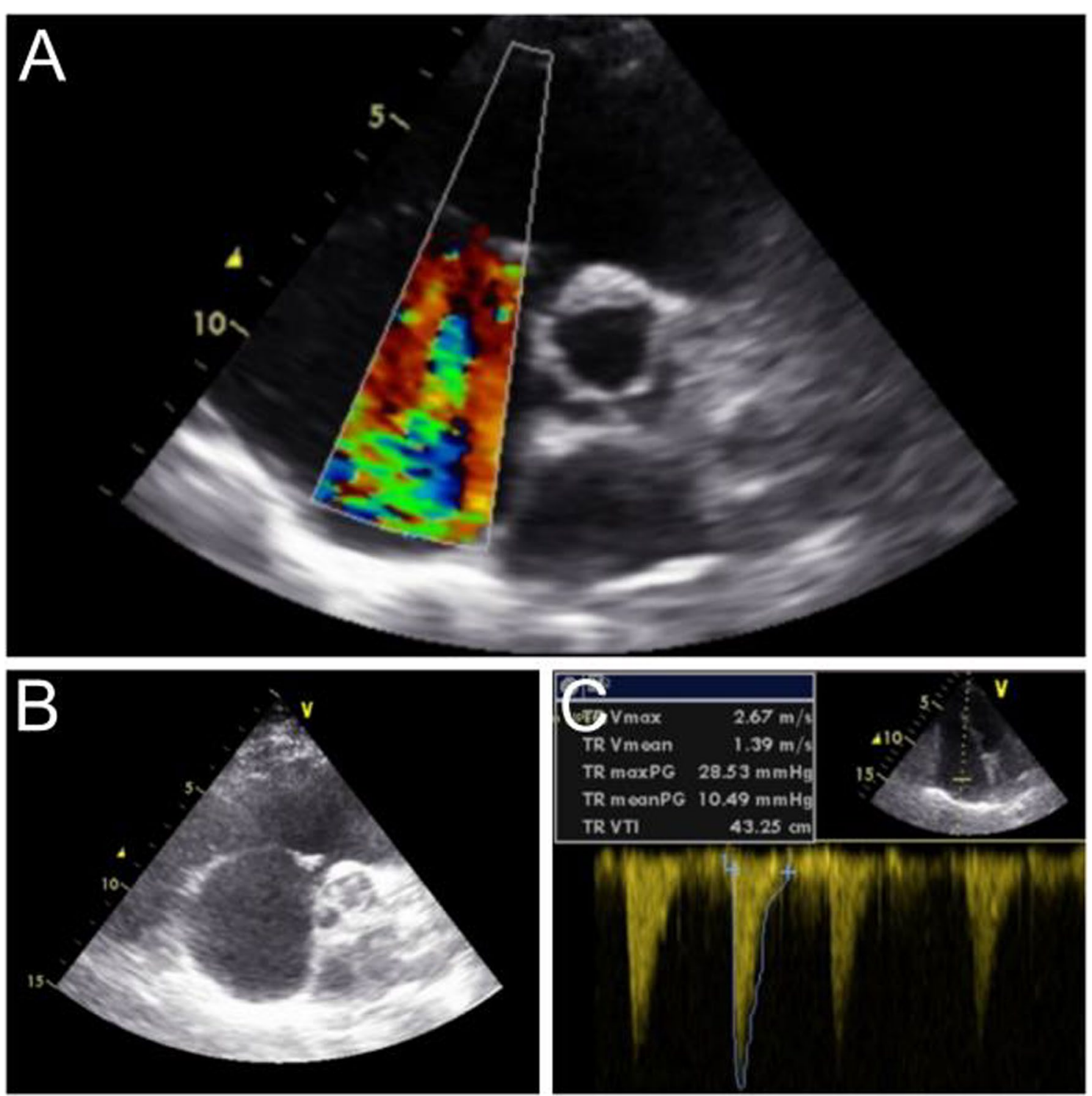

Fig. 1 A Transthoracic echocardiogram (TTE) in parasternal short axis view with evidence of severe tricuspid regurgitation. B TTE in parasternal short axis view shows a dilated right heart. C Continuous wave spectral Doppler analysis of the tricuspid regurgitation (TR) jet for assessing pulmonary arterial pressure 
systolic pulmonary arterial pressure (sPAP), a value of $28 \mathrm{mmHg}$ was calculated.

CT angiography subsequently ruled out pulmonary artery embolism. This examination also revealed multiple liver lesions, which we further investigated. Coronary angiography and right heart catheterization were performed first. There was no relevant coronary artery disease, no shunt defect, and slightly increased pulmonary artery pressure. Further internal and oncological diagnostics initially failed to detect a primary tumor as cause of the liver lesions. Therefore, a biopsy was performed with histological evidence of a well-differentiated G1 NET with high expression of synaptophysin and low co-expression of CD-56. The proliferation rate was $1 \%$, validated with a MIB-1 marker.

In a 24-hour urine collection, the concentrations of 5-hydroxyindoleacetic acid and serotonin were both significantly elevated $(72 \mathrm{mg} / 24$ hours at an upper limit of $8 \mathrm{mg} / 24$ hours and $277 \mu \mathrm{g} / 24$ hours at an upper limit of $240 \mu \mathrm{g} / 24$ hours, respectively). Serotonin levels in serum analysis were normal; however, preceding intake of pantoprazole may result in lowered serum serotonin levels. We assessed the findings as indicative of carcinoid syndrome and investigated cardiac involvement on magnetic resonance imaging (MRI), which showed severely dilated right heart cavities (Fig. 2). The right ventricular myocardial structure showed a hypertrabecularization. The tricuspid valve was rigid and fixed with incomplete closure. The other valves, especially the pulmonary valve, had no pathology. Cine Magnetic Resonance Imaging documented a severe systolic jet from the right ventricle into the right atrium caused by incomplete closure of the tricuspid valve.

The case was discussed interdisciplinarily with cardiologists, cardiothoracic surgeons, and oncologists. The tumor entity was judged to be associated with a good prognosis owing to its slow proliferation rate. Drug treatment was initiated with intramuscular injection of somatostatin. The right heart pathology was deemed to be most relevant regarding symptoms and prognosis. After joint discussion, there was agreement on a protocol involving two octreotide cycles followed by mechanical tricuspid valve replacement. The liver metastases with diameters up to $5 \mathrm{~cm}$ were treated conservatively.

The decision for a mechanical heart valve was made due to the lower risk of fibrosis compared with biological valve replacement. The patient was compliant and agreed to long-term strict [international normalized ratio (INR) 3.0-3.5] oral anticoagulation with phenprocoumon. The operation was performed 5 months after the first hospitalization in June 2015. A $31 \mathrm{~mm}$ Carbomedics heart valve was implanted. The MRI and echo findings were confirmed in situ. The tricuspid valve was found to be rigid and retracted with no possibility of valve reconstruction. In the postoperative course, a hemopericardium occurred with the need for surgical treatment as well as the first onset of typical atrial flutter, which was successfully treated with amiodarone. The patient was discharged 16 days after the tricuspid valve replacement.

In October 2017, the patient presented with recurrent typical atrial flutter and dyspnea. Regarding carcinoid heart disease with an otherwise good postoperative
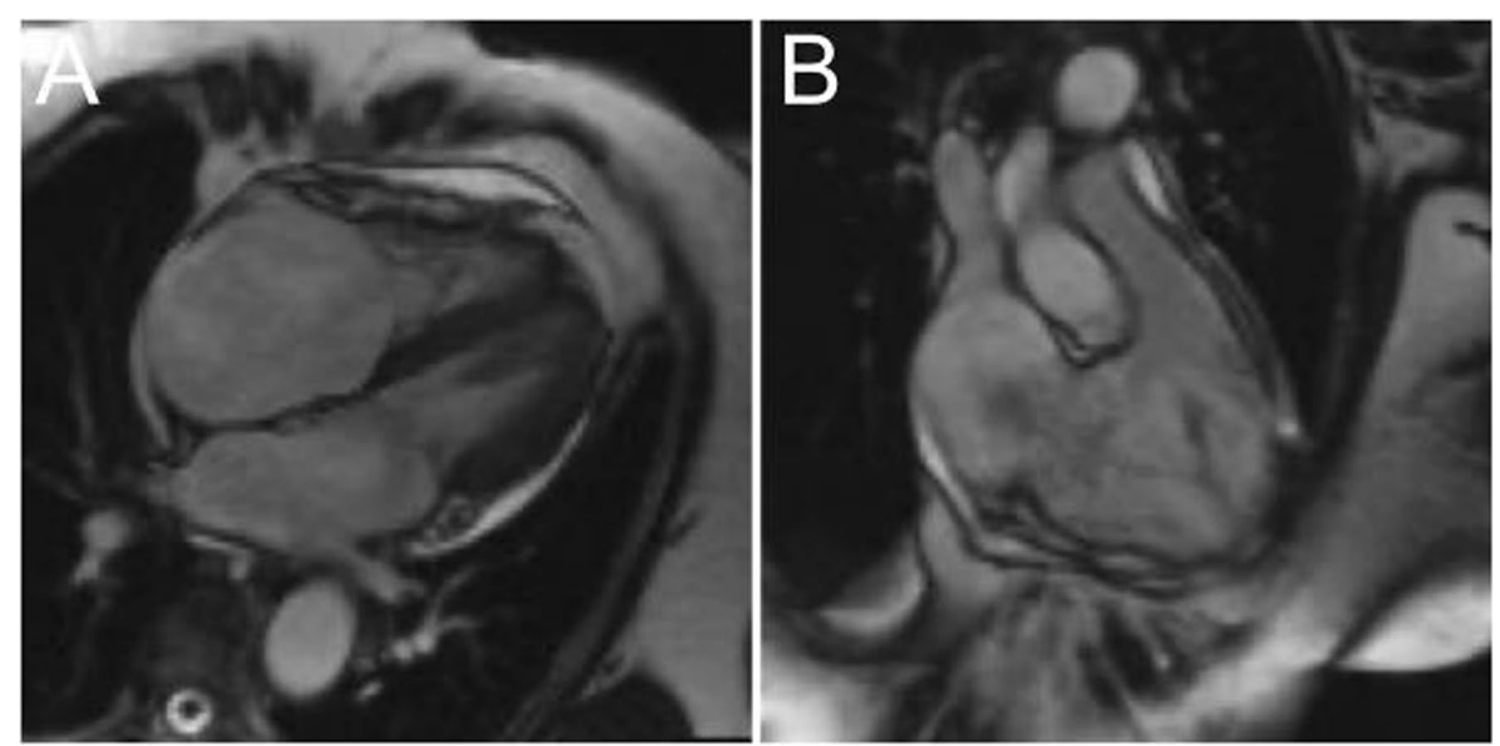

Fig. 2 A MRI scan in four-chamber-view showing a highly dilated right atrium and a hypertrabeculized right ventricle. B MRI scan of the right ventricular outflow tract demonstrating the severe tricuspid regurgitation 
course, we recommended ablation therapy owing to its good long-term efficacy [2]. Twelve-lead-ECG suggested a counterclockwise, cavotricuspid isthmus-dependent atrial flutter with 2:1 conduction and a ventricular rate of 130 beats per minute.

However, the presence of incisional or other types of scar-related atrial flutter was also considered. Invasively, the suspected tachycardia mechanism was confirmed by both entrainment and activation mapping (Fig. 3). After registration and superimposition of the fluoroscopy data, the position of the mechanical valve prosthesis was carefully marked by annotating the locations of the metal artifact recordings of the ablation catheter. Ablation was performed with an inferior cavotricuspid isthmus line leading to termination of the typical atrial flutter. The bidirectional block of the cavotricuspid isthmus was finally confirmed by differential pacing.

The patient completed a follow-up of 3 years after ablation and remains in good clinical condition. All available ECG recordings show sinus rhythm. No new arrhythmias have been noted. Concerning heart failure, she is asymptomatic [New York Heart Association (NYHA) I] under appropriate guideline-directed medication. As such, there is currently no evidence for a progression of the carcinoid heart disease.

\section{Discussion}

Carcinoid heart disease (CHD) is a serious organ manifestation of patients with carcinoid syndrome and advanced neuroendocrine tumor stage, which lowers the 3 -year survival rate to $31 \%[3,4]$. It was first described in 1954 [5]. Approximately 20-50\% of patients with carcinoid syndrome are affected by CHD [6] also known as Hedinger syndrome. Characterized by severe right-sided valvular abnormalities, the disease is caused by enhanced fibrogenesis primarily triggered by serotonin (5-hydroxytryptamine) secreted by neuroendocrine tumors (NET). These NET typically arise in the gastrointestinal and genitourinary tracts. The incidence of carcinoid tumors is $1-2 / 100,000$, with the vast majority of these tumors (>90\%) being benign [6]. Symptomatic CHD usually presents between 50 and 70 years of age [1]. Our patient, who was a 77-year-old lady at initial diagnosis, initially presented to us with signs of heart failure caused by severe tricuspid regurgitation, which is one of the major features of CHD patients. Diarrhea, skin flushing, or bronchospasm were not seen as typical symptoms of carcinoid syndrome in this case [6]. Because serotonin is not activated in the lungs, leftsided CHD is a rarity [1]. Arrhythmias are another very rare complicating feature of this disease; to date, only a single case of typical atrial flutter has been described as an initial manifestation of CHD [7]. Case reports of cardiac arrhythmias in CHD have been described predominantly in the context of carcinoid crisis, a life-threatening condition in which large amounts of serotonin and other vasoactive hormones are released during surgery or chemotherapy. Besides carcinoid crisis, few cases of atrial fibrillation, ventricular tachycardia, and premature ventricular contractions (PVC) have been published [8-10]. One study so far has
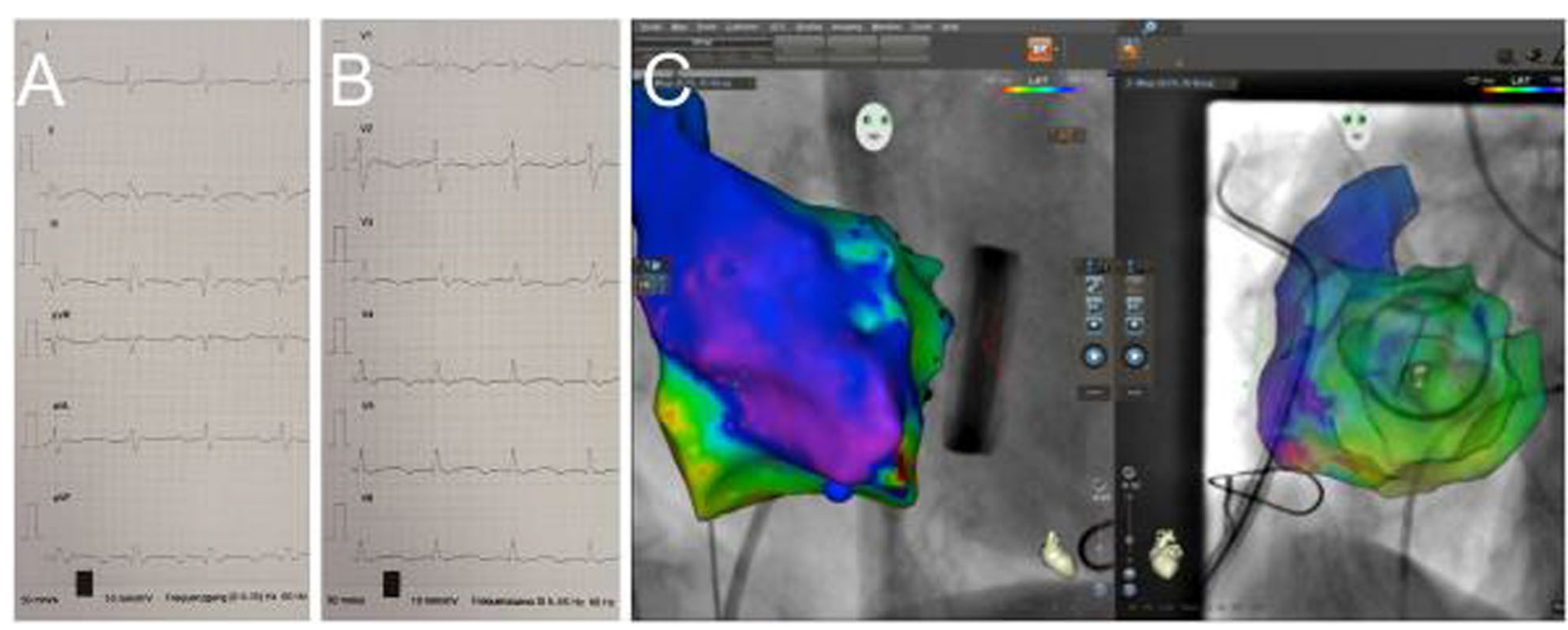

Fig. 3 ECG during typical atrial flutter with limb leads (A) and chest leads (B). C Electroanatomic reconstruction of the right atrium with Local activation time (LAT) mapping visualizing the counterclockwise typical atrial flutter (CARTO 3, Biosense Webster). Fluoroscopic images are integrated into the 3D map using CARTO UNIVU. Black dots mark sites of metal artifact recordings initiated by contact of the ablation catheter with mechanical valve prosthesis (left: right anterior oblique (RAO) $30^{\circ}$, right: left anterior oblique (LAO) $45^{\circ}$ view) 
demonstrated the feasibility of catheter ablation of atrial and ventricular tachyarrhythmias in a cohort of 17 patients with neuroendocrine tumors. Only two of these patients had carcinoid heart disease, and their treated arrhythmia has not been described [11].

In addition to right heart failure and valvular heart disease as causes of arrhythmia, serotonin can increase sympathetic activity and cardiac excitability, triggering these heart rhythm disorders [8]. In our case, typical atrial flutter occurred after successful surgical replacement of the tricuspid valve and complicated the postoperative course. After initial anti-arrhythmic therapy with amiodarone, the atrial flutter recurred. In the early postoperative period, triggers such as inflammation, fluid disturbances, and electrolyte shifts likely triggered the arrhythmia in addition to carcinoid heart disease with right atrial changes. When managed conservatively, typical atrial flutter has a high recurrence rate due to the anatomically fixed structures contributing to the reentrant circuit. Postoperative scarring as has been described for Ebstein anomaly after a tricuspid valve replacement did not lead to additional scar-related atypical right atrial flutter in this case, but possibly retriggered the typical isthmus-dependent atrial flutter by conduction delays. Radiofrequency ablation 2 years after the patient's initial presentation at our clinic made it possible to achieve freedom from recurrence that persisted up to now. Our group recently published that early postoperative ablation of typical flutter in a mixed cardiosurgical cohort was associated with a favorable short- and long-term safety and efficacy profile and could be considered as part of the cardiac rhythm management options in this setting [12]. Early interventional therapy with a high success rate might thus have been an option for our patient in the early postoperative phase, as well.

With an overall poor prognosis of the disease, early identification of the underlying disease was crucial for our patient. The treatment focused on controlling the underlying carcinoid syndrome by initiation of treatment with somatostatin analog octreotide to decrease serotonin levels and consecutive surgical treatment of the severe tricuspid regurgitation [13]. Today, other drug therapy options are available for disease resistant to somatostatin, such as pasireotide or lutetium dotatate [14, 15]. We abstained from liver-directed therapies owing to the well-differentiated G1 NET of our patient with a low proliferation rate of $1 \%$ and the stable course of the disease after successful rhythm control.

The challenge in dealing with carcinoid heart patients is to provide a multidisciplinary and structured therapy, which was performed successfully in this case.

\section{Conclusion}

Research on rhythm management in patients with carcinoid heart disease (CHD) is limited. Typical atrial flutter repeatedly complicated the postoperative course of our patient with CHD and could finally be treated curatively by ablation. RF ablation should be considered as a valuable therapeutic component in the challenging therapy of this disease.

\section{Abbreviations \\ CHD: Carcinoid heart disease; PVT: Deep vein thrombosis; NEC: Neuroendo- crine carcinomas; NEN: Neuroendocrine neoplasms; NET: Neuroendocrine tumors; NYHA: New York Heart Association; PVC: Premature ventricular con- tractions; SPAP: Systolic pulmonary arterial pressure.}

\section{Acknowledgements}

The authors would like to acknowledge all those involved in the management of the patient.

\section{Authors' contributions}

$\mathrm{SG}, \mathrm{GR}$, and $\mathrm{MB}$ conceived the idea of reporting the case. SG and MB drafted the manuscript. RW, BB, GR, RT, and LI contributed to the review and editing of the manuscript. All authors were involved in the clinical management of the patient. All authors read and approved the final manuscript.

\section{Funding}

There was no funding.

\section{Availability of data and materials}

Case data derive from patient's records and are therefore not publicly available.

\section{Declarations}

Ethical approval and consent to participate Not applicable.

\section{Consent for publication}

Written informed consent was obtained from the patient for publication of this case report and any accompanying images. A copy of the written consent is available for review by the Editor-in-Chief of this journal.

\section{Competing interests}

The authors declare that they have no competing interest to declare.

Received: 12 October 2021 Accepted: 30 December 2021

Published online: 02 February 2022

\footnotetext{
References

1. Jin C, et al. Carcinoid heart disease: pathophysiology, pathology, clinical manifestations, and management. Cardiology. 2021;146(1):65-73.

2. Kuck KH, Phan HL, Tilz RR. New ESC guidelines 2019 for the treatment of supraventricular tachycardia. Herz. 2019;44(8):701-11.

3. Oleinikov $\mathrm{K}$, et al. Update in carcinoid heart disease - the heart of the matter. Rev Endocr Metab Disord. 2021;22:553.

4. Dashwood A, Rahman A, Pavicic M. Carcinoid heart disease. Eur Heart J. 2015;36(34):2326

5. Thorson A, et al. Malignant carcinoid of the small intestine with metastases to the liver, valvular disease of the right side of the heart (pulmonary stenosis and tricuspid regurgitation without septal defects), peripheral vasomotor symptoms, bronchoconstriction, and an unusual type of cyanosis; a clinical and pathologic syndrome. Am Heart J. 1954:47(5):795-817.
} 
6. Dobson $\mathrm{R}$, et al. The clinical presentation and management of carcinoid heart disease. Int J Cardiol. 2014;173(1):29-32.

7. Fragkou PC, et al. Rare presentation of a metastatic pancreatic neuroendocrine neoplasm presenting with atrial flutter. Eur J Case Rep Intern Med. 2021;8(1):002216.

8. Langer C, et al. Atrial fibrillation in carcinoid heart disease: the role of serotonin. A review of the literature. Clin Res Cardiol. 2007;96(2):114-8

9. Kuczma JA, Grzywa M. Broncho-pulmonary carcinoid with the cardiac arrhythmia manifestation. Pol Arch Med Wewn. 2006;116(4):971-3.

10. Rupp $A B$, et al. Carcinoid syndrome-induced ventricular tachycardia. Case Rep Cardiol. 2016;2016:9142598.

11. Shabtaie SA, et al. Catheter ablation in patients with neuroendocrine (carcinoid) tumors and carcinoid heart disease: outcomes, peri-procedural complications, and management strategies. JACC Clin Electrophysiol. 2021;7(2):151-60.

12. Iden $\mathrm{L}$, et al. Ablation therapy as management of typical atrial flutter in the early period after cardiac surgery. Cardiol Ther. 2021;10:569.

13. Davar J, et al. Diagnosing and managing carcinoid heart disease in patients with neuroendocrine tumors: an expert statement. J Am Coll Cardiol. 2017:69(10):1288-304.

14. Wolin EM, et al. Phase III study of pasireotide long-acting release in patients with metastatic neuroendocrine tumors and carcinoid symptoms refractory to available somatostatin analogues. Drug Des Dev Ther. 2015;9:5075-86

15. Maqsood MH, Tameez Ud Din A, Khan AH. Neuroendocrine tumor therapy with lutetium-177: a literature review. Cureus. 2019;11(1):e3986.

\section{Publisher's Note}

Springer Nature remains neutral with regard to jurisdictional claims in published maps and institutional affiliations.

- fast, convenient online submission

- thorough peer review by experienced researchers in your field

- rapid publication on acceptance

- support for research data, including large and complex data types

- gold Open Access which fosters wider collaboration and increased citations

- maximum visibility for your research: over 100M website views per year

At BMC, research is always in progress.

Learn more biomedcentral.com/submissions 